\title{
High-Strength Composites Based on Tung Oil Polyurethane and Wood Flour: Effect of the Filler Concentration on the Mechanical Properties
}

\author{
U. Casado, N.E. Marcovich, M.I. Aranguren, M.A. Mosiewicki \\ Institute of Materials Science and Technology (INTEMA), National University of Mar del Plata-National \\ Research Council of Argentina (CONICET), 7600 Mar del Plata, Argentina
}

\begin{abstract}
New composite materials with a high percentage of their composition based on renewable resources, matrix and reinforcement, were prepared. The excellent compatibility of the main phases lead to an unusual outstanding composite behavior as follows: higher modulus and strength, but also higher deformability and fracture resistance than the neat polymer. A biobased polyol was obtained by chemical modification of tung oil to be used in the production of polyurethane composites reinforced with pine wood flour (WF). The mechanical behavior as a function of wood flour content was evaluated through tensile and impact tests. Increasing the filler content induced an increase in tensile modulus and strength as well as in impact strength. Composites containing 10 and 15 wt $\%$ of wood flour presented higher tensile ultimate strain than that of the unfilled polyurethane. These results were explained by a strong interfacial adhesion (physical and chemical bonding), which was also supported by SEM micrography and strength modeling. POLYM. ENG. SCI., 49:713721, 2009. () 2009 Society of Plastics Engineers
\end{abstract}

\section{INTRODUCTION}

The new trend in the polymer composite industry and academy is the search for alternative products that can be prepared from renewable resources, offering also the possibility to decompose by biodegradation. In the plastic industry, the polyurethanes are one of the most required polymers due to their versatility. The synthesis of polyurethanes involves the reaction of a component containing isocyanate groups and another one containing hydroxyl groups, both with functionalities equal or higher than two [1]. In general, the polyurethane industrial production is carried out with

Correspondence to: M.A. Mosiewicki; e-mail: mirna@fi.mdp.edu.ar Contract grant sponsor: National Research Council of Argentina (CONICET); contract grant number: PIP 6250/05; contract grant sponsor: Science and Technology National Promotion Agency-National University of Mar del Plata (ANPCyT-UNMdP); contract grant number: PICTO No. 14-381.

DOI 10.1002/pen.21315

Published online in Wiley InterScience (www.interscience.wiley.com) (C) 2009 Society of Plastics Engineers materials derived from the petroleum industry [2]. In the recent decades, there have been several works, which indicate that vegetable oils can be used as the polyol component in polyurethane materials after being modified by the introduction of hydroxyl groups in their structure [3, 4]. Thus, the rather inexpensive and widely available natural oils become interesting raw materials.

Oils are triglyceride molecules composed of saturated and unsaturated fatty acids. These oils can be functionalized by hydroxylation of the carbon-carbon double bonds with a peroxy acid and/or by alcoholysis with a triol like glycerol or triethanolamine to reach a hydroxyl value useful for elastomeric or rigid polyurethane production [3-5].

Tung oil is obtained from the seeds or nuts of the tung tree. It is composed mainly by $\alpha$-eleostearic acid (around $80 \%$ ) with three conjugated double bonds (9-10 cis, 1112 trans, 13-14 trans) and minor percentages of oleic acid (one double bond) and linolenic acid (three nonconjugated double bonds) [6]. The high amount of carbon-carbon double bonds in the chemical structure of tung oil is the reason for having fast polymerization in presence of oxygen and for being one of the most used oils (together with linseed oil) in the paint and varnish industry [7].

Although any oil can break down by alcoholysis, which increases the $\mathrm{OH}$ value, tung oil, with multiple carboncarbon double bonds, can also be modified by hydroxylation increasing even more the $\mathrm{OH}$ concentration in the product.

On the other hand, the incorporation of low cost natural fibers obtained from a sustainable resource in polymeric matrices widens the use of these materials to new different applications. The properties of the composites can change significantly compared with the unreinforced polymer, whereas the cost of the products can be reduced by using natural fibers as reinforcement. In general, the incorporation of fillers to polyurethanes results in higher modulus due to the stiffness of the particles and also higher strength when the interface fiber-matrix is good, but the ultimate strain is reduced. Recently, a work on nanoparticles reinforcing rubbery polyurethanes has reported an increase in modulus and strength without sacrificing the high deformation at break of the polymer matrix $[8,9]$. 
The multiple interactions between the reinforcing particles and the thermoset matrix, coupled to the nature of the interphase region, make the response of the composite materials extremely complex. The mechanical behavior of the new composites depends of a series of factors besides the individual constituent phases, such as interfacial region, size, shape, aspect ratio and distribution of the reinforcing particles, presence of voids, etc. Besides the typical tensile tests to evaluate mechanical behavior, the impact test can be a useful tool to complement the information of this new material because the absorption capacity of energy is highly sensitive to the interface and the filler concentration [10].

The development and analysis of new and existing mathematical models for the prediction of the mechanical behavior of particulate and fiber reinforced traditional composites has been the focus of many works in the literature. However, fewer works can be found reporting similar finding in the area of composites based in natural raw materials [11].

In this work, the preparation of a polyurethane using a polyol synthesized from tung oil and reinforced with wood flour particles is reported. The densities of the materials were determined to corroborate that the composites were compact structures. The response of the materials in tensile and impact tests was evaluated. The mechanical properties were analyzed using simple models.

\section{Theoretical Approach}

The modeling of the mechanical behavior of reinforced materials can help in organizing the analysis and evaluation of new products. The simplest equations to model the mechanical behavior of an elastic system formed by two components correspond to the parallel and series models that consider iso-strain and iso-stress, respectively [1214]. Other simple models for predicting the modulus of the composite $\left(E_{\mathrm{c}}\right)$ as a function of the filler volume fraction $\left(V_{\mathrm{f}}\right)$ in a compact material without voids have been carefully organized by Ahmed and Jones in their review work [12].

The assumptions and mathematical equations of several simple models used in the prediction of the modulus of composites with different filler content are the following:

Parallel model (upper bound) assumes uniform strain in both phases

$$
E_{\mathrm{c}}=E_{\mathrm{m}} \cdot V_{\mathrm{m}}+E_{\mathrm{f}} \cdot V_{\mathrm{f}}
$$

Series model (lower bound) assumes uniform stress in both phases

$$
E_{\mathrm{c}}=\frac{E_{\mathrm{f}} \cdot E_{\mathrm{m}}}{E_{\mathrm{f}} \cdot V_{\mathrm{m}}+E_{\mathrm{m}} \cdot V_{\mathrm{f}}}
$$

Hirsch model proposes a weighed average of Eqs. 1 and 2 to take in account the complex stress distribution in the individual phases. The parameters $x$ and $(1-x)$ are the relative proportions of the material conforming to the upper and lower bound solutions.

$$
E_{\mathrm{c}}=x \cdot\left(E_{\mathrm{m}} \cdot V_{\mathrm{m}}+E_{\mathrm{f}} \cdot V_{\mathrm{f}}\right)+(1-x) \frac{E_{\mathrm{f}} \cdot E_{\mathrm{m}}}{E_{\mathrm{f}} \cdot V_{\mathrm{m}}+E_{\mathrm{m}} \cdot V_{\mathrm{f}}}
$$

Halpsin-Tsai is applied to reinforcements of diverse shapes. $\xi$ is a parameter that serves to adjust the modulus between the maximum value corresponding to the fiber and the minimum of the matrix.

$$
\begin{gathered}
E_{\mathrm{c}}=E_{\mathrm{m}} \cdot\left(\frac{1+\xi \cdot \chi \cdot V_{\mathrm{f}}}{1-\chi \cdot V_{\mathrm{f}}}\right) \\
\chi=\frac{E_{\mathrm{f}}-E_{\mathrm{m}}}{E_{\mathrm{f}}+\xi \cdot E_{\mathrm{m}}}
\end{gathered}
$$

$E_{\mathrm{f}}$ and $E_{\mathrm{m}}$ are the modulus of the fiber and matrix, respectively.

Useful models for the prediction of the strength of filled systems have been less successful [12]. One of the few simple models is due to Pukanszky et al. [15] who assumed that the yield stress of particulate filled polymer composites is determined by the decrease of the effective load bearing cross section of the matrix due to filling and by the polymer-filler interaction. The proposed equation is as follows:

$$
\sigma_{c}=\sigma_{m}\left(\frac{1-V_{f}}{1+2.5 \cdot V_{f}}\right) \cdot \exp \left(B \cdot V_{f}\right)
$$

where, $B$ is a parameter characterizing the interfacial interaction filler-matrix that can be used to quantify the reinforcement efficiency of the filler. If $B=0$ the filler acts as a void and no adhesion and no stress transfer take place at the filler-matrix interface. For $B>3$ the filler-matrix interface is good and a reinforcing effect is obtained.

\section{EXPERIMENTAL}

\section{Materials}

The natural polyol was obtained from tung oil supplied from Cooperativa Agrícola Limitada de Picada Libertad, Argentina (saponification value $=223 \mathrm{mg} \mathrm{KOH} / \mathrm{g}$, acid number $=2.11 \mathrm{mg} \mathrm{KOH} / \mathrm{g})$. Hydrogen peroxide $(30$ $\mathrm{wt} \%$ ) and formic acid (88 wt\%) from Laboratorios Cicarelli were used in the hydroxylation reaction. Triethanolamine $(>99 \%)$ from Laboratorios Cicarelli and lithium hydroxide $(>99 \%)$ from Fluka were used in the alcoholysis reaction. Tetrahydrofurane (THF) from Fluka was used to decrease the initial viscosity of the polyol.

For the preparation of rigid polyurethanes, the isocyanate used was $4,4^{\prime}$-diphenylmethane diisocyanate (pMDI) 
prepolymer (Rubinate 5005, Huntsman Polyurethanes) with an equivalent weight of $131 \mathrm{~g} / \mathrm{eq}$. The modified oil obtained after the two modification steps was used as a natural hydroxyl source in the polyurethane production and the hydroxylated tung oil (HTO) product of the hydroxylation reaction is just an intermediate product in this work.

Pine wood flour (Jorge Do Santos Freire, Buenos Aires, Argentina) with particle sizes $\leq 64 \mu \mathrm{m}$ was used as the reinforcement/reactive filler.

Wood flour and synthesized polyol were dried at $70^{\circ} \mathrm{C}$ until constant weight in a vacuum oven previously to be used.

A back titration technique was used to determine the $\mathrm{OH}$ value of the wood flour $(233.3 \mathrm{mg} \mathrm{KOH} / \mathrm{g})$. A measured weight of wood flour was mixed with excess of pMDI. After the reaction was completed, the free NCO groups were determined by a standard method [16].

\section{Synthesis of Tung Oil Based Polyols}

Hydroxylated Tung Oil. Hydrogen peroxide solution and formic acid were added in a stirred reactor and the temperature was raised to $40^{\circ} \mathrm{C}$ and maintained during the rest of the reaction. During this initial step, the hydrogen peroxide reacts with the formic acid to form performic acid. Then, the oil was added drop wise and the reaction allowed to proceed for $3 \mathrm{~h}$. Ratios oil $/ \mathrm{H}_{2} \mathrm{O}_{2}$ (weight) $=$ $3.5 / 1$ and $\mathrm{H}_{2} \mathrm{O}_{2} / \mathrm{HCOOH}$ (weight) $=1 / 1.9$ were used. As the tung oil is incorporated, it undergoes an intermediate process of epoxidation. The epoxide group is unstable under the strong acid conditions and it opens to form hydroxyl groups [4]. After which the final product was cooled down to room temperature, and then it was separated in two layers. The upper layer was directly distilled under vacuum to eliminate the remaining water and acid.

Alcoholysed Hydroxilated Tung Oil. The hydroxylated tung oil, dry triethanolamine, and lithium hydroxide (catalyst) were added together in a mechanically stirred reactor. The temperature was raised to $150^{\circ} \mathrm{C}$ in $0.5 \mathrm{~h}$ and maintained at this value for the following $2.5 \mathrm{~h}$. The hydroxylated tung oil/triethanolamine weight ratio was of $2 / 1$ and the catalyst was used in a $0.2 \mathrm{wt} \%$ of the total reactants. This step of reaction is basically a transesterification process where the exchange of the alkoxy group of an ester compound by another alcohol takes place. The final product is a mixture of different alcohols containing one or two hydroxylated fatty acid chains with an average hydroxyl number of $434 \mathrm{mg} \mathrm{KOH} / \mathrm{g}$.

\section{Polyurethane Production}

The index (equivalents $\mathrm{NCO} /$ equivalents $\mathrm{OH}$ ) used was 1.1. Both, hydroxyl groups coming from the polyol and from wood flour were considered in the calculation of this index. The modified polyol (previously dried) was dissolved in THF (weight ratio $=1 / 1$ ) to decrease the initial viscosity and to reduce the reaction rate. After that, the pMDI (or pMDI plus filler, for reinforced polyurethanes) was added and the system was mechanically mixed for $20 \mathrm{~s}$. Then the reactive mixture was put in a metal mold of $14 \mathrm{~cm}$ of internal diameter. The THF was allowed to evaporate, and then the mold was closed and the sample cured at $75^{\circ} \mathrm{C}$ and $4 \mathrm{MPa}$ for $1 \mathrm{~h}$. Using this procedure, plates of $1 \mathrm{~mm}$ thickness and containing $0,10,15,20$, and $30 \mathrm{wt} \%$ of wood flour were obtained.

\section{Characterization}

Density of the Materials. The composite densities were determined by picnometry over three specimen of each sample and were compared with the theoretical values calculated from the following rule of mixture:

$$
\frac{1}{\rho_{\mathrm{c}}}=\frac{W_{\mathrm{m}}}{\rho_{\mathrm{m}}}+\frac{W_{\mathrm{f}}}{\rho_{\mathrm{f}}}
$$

where, $\rho$ is the density and $W$ is the weight fraction, and the subscripts $\mathrm{c}, \mathrm{m}$, and $\mathrm{f}$ correspond to composite, matrix, and filler, respectively. The value of $\rho_{\mathrm{m}}=1.24 \mathrm{~g} /$ $\mathrm{cm}^{3}$ was experimentally determined, and $\rho_{\mathrm{f}}$ was taken as the density value of the wood flour cell walls, $1.53 \mathrm{~g} / \mathrm{cm}^{3}$ [17], because the particles are compressed in the composite and the resin is filling the cell lumens [18].

From the predicted densities (Eq. 7) and the experimental data, the nominal volume fraction of filler $\left(V_{\mathrm{f}}\right)$ and matrix $\left(V_{\mathrm{m}}\right)$ in the composite can be calculated. Thus, assuming that the component volumes can be added to obtain the composite volume and considering that no voids are present in the samples, the nominal volume fractions become as following:

$$
\begin{gathered}
V_{\mathrm{f}}=\frac{v_{\mathrm{f}}}{v_{\mathrm{c}}}=\frac{\frac{W_{\mathrm{f}}}{\rho_{\mathrm{f}}}}{\frac{W_{\mathrm{f}}}{\rho_{\mathrm{f}}}+\frac{W_{\mathrm{m}}}{\rho_{\mathrm{m}}}} \\
V_{\mathrm{m}}=\frac{v_{\mathrm{m}}}{v_{\mathrm{c}}}=\frac{\frac{W_{\mathrm{m}}}{\rho_{\mathrm{m}}}}{\frac{W_{\mathrm{f}}}{\rho_{\mathrm{f}}}+\frac{W_{\mathrm{m}}}{\rho_{\mathrm{m}}}}
\end{gathered}
$$

Microscopy. Scanning Electron Microscopy (SEM) was used to obtain photographs of the fracture surfaces of the composites tested in tensile and impact tests (scanning electron microscope Philips model SEM 505). Microscopy specimens were coated with gold before the analysis.

Dynamic-Mechanical Tests. Dynamic-mechanical tests were performed in a Perkin Elmer dynamic mechanical analyzer, DMA 7e, using tensile fixture under nitrogen atmosphere. The specimens were cut to $20 \mathrm{~mm} \times 4 \mathrm{~mm} \times$ $1 \mathrm{~mm}$, linear dimensions, and measured $\pm 0.01 \mathrm{~mm}$. The dynamic and static stresses were kept at 200 and $240 \mathrm{KPa}$, respectively. The frequency of the forced oscillations was fixed at $1 \mathrm{~Hz}$ and the heating rate was of $10^{\circ} \mathrm{C} / \mathrm{min}$. At 
least, two replicate determinations were made for each sample.

Tensile Tests. Specimens of each sample were cut and tested at room temperature at $1 \mathrm{~mm} / \mathrm{min}$ in an INSTRON 8501 Universal testing machine, according to the ASTM D638-94. At least, four specimens of each sample were used, and the average values are reported.

Instrumented Falling Weight Impact Tests. Instrumented dart impact testing was performed using a falling weight impact machine (Fractovis Ceast 6789). Test plates of $1 \mathrm{~mm}$ thickness were clamped on an annular stainless steel ring with an internal diameter of $40 \mathrm{~mm}$. The samples were impacted with a hemispherical tipped dart at an incident speed of $2 \mathrm{~m} / \mathrm{s}$ using an impactor mass of $19.8 \mathrm{~kg}$. The incident energy was sufficient for assuring that the impact test was performed to fracture. Sets of four discs of each sample were tested.

The impact force $(F)$-displacement $(x)$ traces were recorded to determine the following impact properties:

The impact strength $\left(\sigma_{\mathrm{d}}\right)$ related to crack initiation was computed converting the maximum registered load $\left(F_{\max }\right)$ to stress with the use of the following equation:

$$
\sigma_{\mathrm{d}}=2.5 \cdot \frac{F_{\max }}{b^{2}}
$$

where $b$ is the sample thickness.

The total energy $\left(E_{\mathrm{t}}\right)$ required to complete penetration and fracture in the specimen was calculated as the total area under the load-displacement curve normalized by the thickness:

$$
E_{\mathrm{t}}=\frac{1}{b} \int_{0}^{X_{\text {total }}} F d x
$$

The initiation energy $\left(E_{\mathrm{i}}\right)$ is the energy required to initiate the crack and it was calculated as the area under the load-displacement curve just up to the maximum load, normalized by thickness:

$$
E_{\mathrm{i}}=\frac{1}{b} \int_{0}^{X_{\max }} F d x
$$

The propagation energy $\left(E_{\mathrm{p}}\right)$ is the energy required to complete disk penetration and it was calculated as the area under the load-displacement curve after the maximum load, normalized by the thickness:

$$
E_{\mathrm{p}}=\frac{1}{b} \int_{X_{\max }}^{X_{\text {total }}} F d x
$$

The ductility index (DI) was also calculated as the ratio of the propagation energy, $E_{\mathrm{p}}$, to the total energy, $E_{\mathrm{t}}$, as follows:

$$
\mathrm{DI}=\frac{E_{\mathrm{t}}-E_{\mathrm{i}}}{E_{\mathrm{t}}}=\frac{E_{\mathrm{p}}}{E_{\mathrm{t}}}
$$

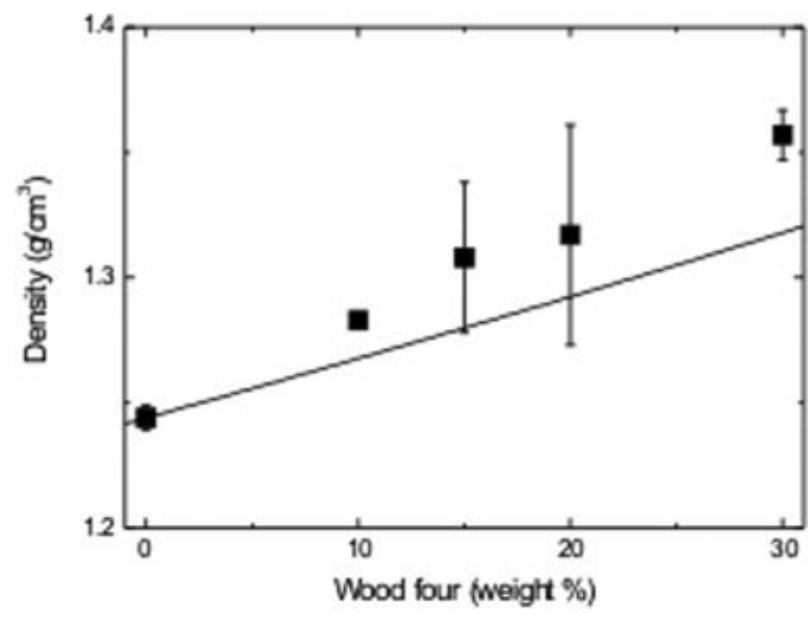

FIG. 1. Experimental and theoretical densities for the composites.

\section{RESULTS AND DISCUSSION}

The relationship between the reinforced polyurethane and density is illustrated in Fig. 1. Increasing the filler content increases the density. The experimental values (symbols) were higher than the theoretical ones (continuous line, Eq. 7), probably due to the strong interaction between polyurethane and wood flour that lead to a compact material, with more crosslinking points (introduced by reaction with the wood flour) than those present in the unfilled polyurethane. Both features lead to more compact and dense materials.

\section{Morphological Analysis}

Figure 2 shows the fracture surface of rigid polyurethanes formulated with the natural polyol [Alcoholysed Hydroxilated Tung Oil (AHTO)] without reinforcement (Fig. 2A) and with the incorporation of WF (Fig. 2B-E). It can be observed that the matrix is not foamed in any case. As it was expected, the surface of the reinforced materials is substantially more irregular than the fracture surface of the neat polyurethane.

Figure 2B shows the fracture surface micrograph at $100 \times$ magnification of an impact tested sample containing $20 \mathrm{wt} \%$ WF. It is clear that the particles are well-dispersed into the matrix and the sample presents a homogeneous pattern of fracture with no pull out. The observation of one of the WF short fibers at higher magnification (Fig. 2C) denotes that the failure of the fibers occur at the same level as the matrix without appreciable damage in the fiber-matrix interfacial region. This behavior is indicating that there is excellent fiber-matrix adhesion at the interfacial region. The $\mathrm{OH}$ groups in the $\mathrm{WF}$ can react with the pMDI originating strong covalent bonds between fibers and matrix. The fracture surfaces of the broken samples during tensile testing also lead to the same conclusion, as can be concluded from the observation of Fig. 2D and E. Additionally, Fig. 2D and E show tensile frac- 

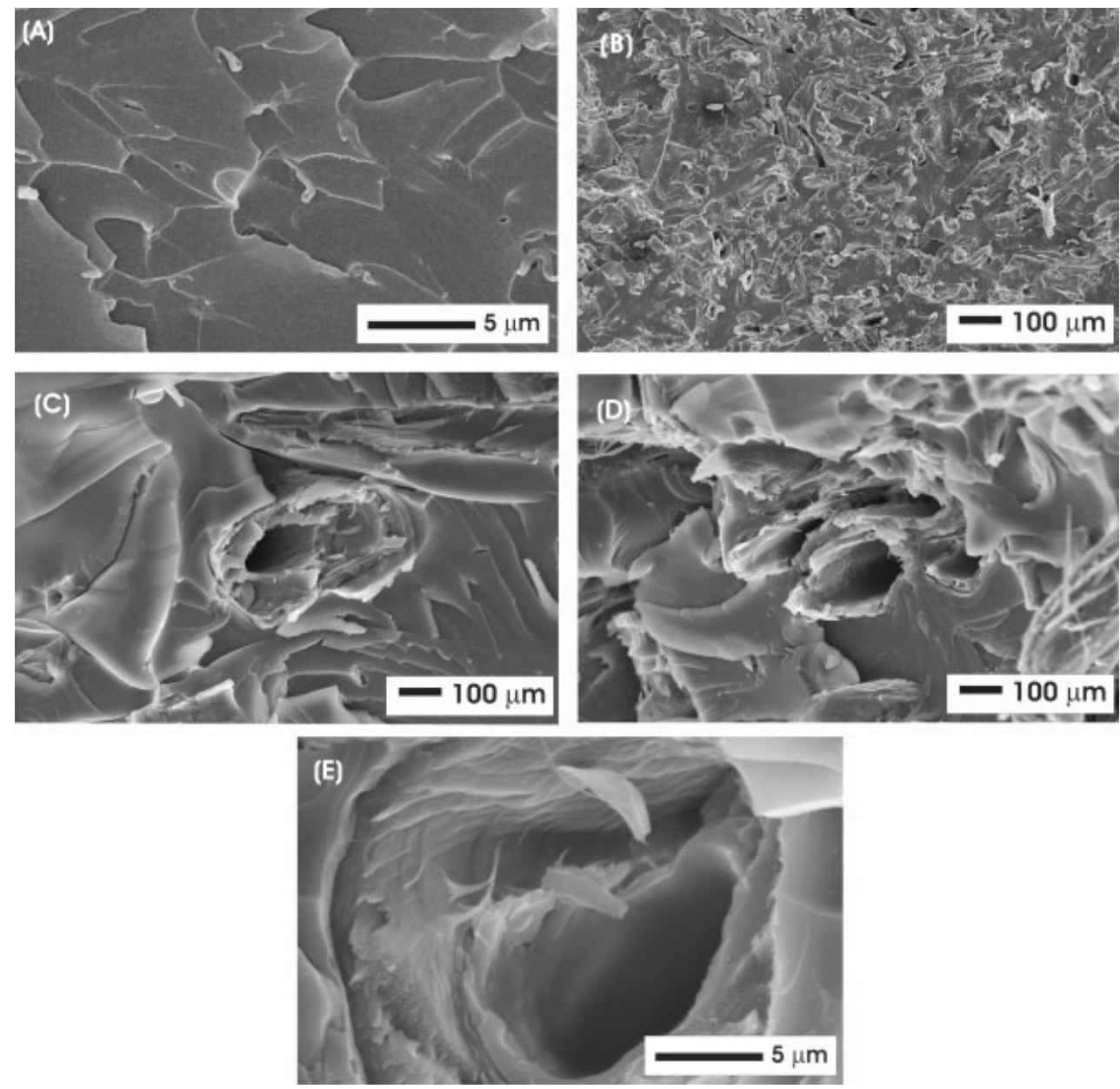

FIG. 2. SEM micrographs of fragile fracture, (A) matrix, (B) impact tested composite with $20 \mathrm{wt} \%$ of WF ( $\times 100)$, (C) impact tested composite with $20 \mathrm{wt} \%$ of WF $(\times 1000)$, (D) tensile tested composite with 10 wt $\%$ of WF $(\times 1000)$, and (E) tensile tested composite with $10 \mathrm{wt} \%$ of WF $(\times 5000)$.

ture surfaces of one sample containing $10 \mathrm{wt} \% \mathrm{WF}$, where it is clear that the failure is not located at the interface, but instead is occurring in the fiber.

On the other hand, the fracture surface of the matrix looks rougher, with more plastic deformation, in the tensile fracture surfaces as compared with the surfaces that result from the impact tests. This is attributed to the higher test speed used in the impact testing, which results in less plastic deformation of the polymer (Fig. 2C and D).

Because of the good wetting of the fiber by the polymer, as can be observed in Fig. 2B-E, it is possible to obtain a composite with up to $30 \mathrm{wt} \%$ of wood flour without voids in its structure. On the other hand, it is difficult to obtain compact reinforced polyurethanes using higher WF contents due to the incomplete wetting of the filler and the high rate of the reaction, which finishes before a good mixing could be achieved.

\section{Dynamical-Mechanical Analysis}

Rigid polyurethanes prepared with different weight percentages of WF were analyzed by DMA (see Fig. 3).
The main relaxation of the polymeric matrix ( $\alpha$ transition related to the peak of $\tan \delta$ ) is quite broad due to the large number of different relaxation modes that can derive from the complex chemical structure of these oil-based polymers [3]. The storage modulus presents a direct functionality with the WF content in the whole range of temperatures (Fig. 3A). A 67\% increase of the room temperature modulus is achievable by the addition of a $30 \mathrm{wt} \%$ WF to the formulation.

As expected, at temperatures above $\alpha$ transition, the differences are more important due to the rigid filler particles that restrict the segmental motion of the rubbery matrix and so the storage modulus is enhanced. In this temperature range, the increase of the storage modulus is of about $500 \%$ for the same $30 \mathrm{wt} \% \mathrm{WF}$ addition (Fig. 3A).

The most interesting observation from this analysis is the displacement of the peak in the $\tan \delta$ curves toward higher temperatures with increasing WF concentration in the composites (Fig. 3B). The temperature of the maximum of the peaks associated to the main relaxation of the matrix is related to the polyurethane glass transition temperature $\left(T_{\mathrm{g}}\right)$. As shown in Fig. 3B, the neat and reinforced 

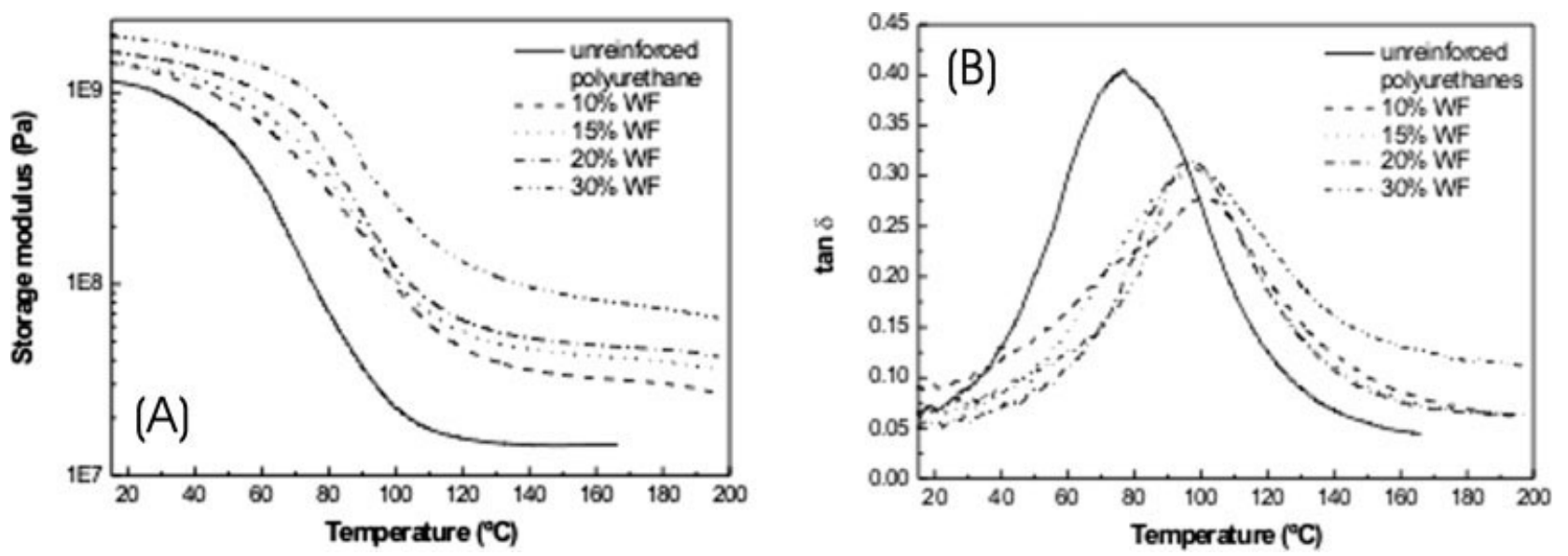

FIG. 3. Dynamical mechanical properties of the matrix and composites. (A) Storage modulus as a function of the temperature, (B) $\tan \delta$ as a function of the temperature.

polyurethanes exhibit one wide peak in the range of temperature analyzed. The width of the peak becomes broader with the WF incorporation due to different relaxation mechanisms appearing in the reinforced materials as a consequence of the added filler. The broadening of the $\tan \delta$ peak is often assumed to be due to a broader distribution in molecular weight between crosslinking points or heterogeneities in the network structures [19].

The important shift toward higher temperatures that occur in the $\tan \delta$ peak $\left(77^{\circ} \mathrm{C}\right.$ and $98^{\circ} \mathrm{C}$ for the neat polyurethane and the $30 \% \mathrm{WF}$ composite, respectively) suggests a strong interaction between the reinforcing filler and the polyurethane matrix. As it was anticipated, the WF presents free hydroxyl groups able to interact chemically with the pMDI and to originate new bonds, chemically linking the matrix and the reinforcement. The reduced height of the peak after WF addition is due to a dilution effect and also to the presence of filler that reduces the composite's ability to dissipate mechanical energy, that is, it reduces the damping capability.

Notice that at WF contents less than $15 \mathrm{wt} \%$, $\tan \delta$ appears as the sum of two peaks, one around $75^{\circ} \mathrm{C}$ (the glass transition temperature of the neat polyurethane) and a new one at $\sim 100^{\circ} \mathrm{C}$. This would be the result of network heterogeneity; some regions in the polymer corresponding to almost unmodified bulk polyurethane and some polyurethane regions affected by the extra crosslinking with the filler. At higher WF percentages, the entire polymer is participating in the network filler-matrix and thus, the low temperature tail of the peak disappears. Notice, however, that there is a larger tailing at higher temperatures, indicating that relaxation of less mobile segments is taking place at these higher temperatures.

\section{Mechanical Properties and Mathematical Validation}

The tensile properties were evaluated as a function of the WF content and the results are presented in Table 1.
The addition of WF lead to a large increase the modulus as it was mentioned above for DMA results. In this case, the Young modulus increases more than three times, respect to that of the neat polyurethane, by adding 30 wt $\%$ WF. The strength also increases with filler concentration. These results are explained by the higher modulus and strength of the wood fibers compared with those of the matrix, in addition to the good adhesion wood-polymer. Overall, the composites showed a marked improvement in modulus and strength relative to the unfilled material. This large improvement is based on the chemical and hydrogen bonds developed between the isocyanate groups of the pMDI and the hydroxyl groups of the wood flour cell walls, as it was already discussed for the large upward shift observed in the $T_{\mathrm{g}}$ of the composites. The filler-matrix bonds act as extra crosslinks at the interfaces of the composite materials, improving the matrix-fiber adhesion, giving more compact structures (density section) as well as enhancing the mechanical properties, modulus and ultimate stress.

The ultimate deformation certainly increases with the incorporation of $10 \mathrm{wt} \%$ of $\mathrm{WF}$, but decreases in a linear way for higher WF contents (see Fig. 4). In fact, composites containing 10 and $15 \mathrm{wt} \%$ of wood flour show an ultimate deformation higher than that of the matrix. In the conventional microcomposites the addition of fibers improves the modulus. Besides, higher strength can also be observed in systems with good interfacial interactions,

TABLE 1. Tensile properties of composites with different wood flour contents.

\begin{tabular}{cccc}
\hline $\begin{array}{l}\text { Wood flour } \\
(\mathrm{wt} \%)\end{array}$ & $\begin{array}{c}\text { Tensile modulus } \\
(\mathrm{GPa})\end{array}$ & $\begin{array}{c}\text { Tensile strength } \\
(\mathrm{MPa})\end{array}$ & $\begin{array}{c}\text { Tensile ultimate } \\
\text { deformation }(\times 1000)\end{array}$ \\
\hline 0 & $0.91 \pm 0.12$ & $26.00 \pm 2.56$ & $34.5 \pm 1.8$ \\
10 & $1.23 \pm 0.13$ & $35.65 \pm 1.75$ & $45.9 \pm 3.5$ \\
15 & $1.80 \pm 0.09$ & $41.90 \pm 1.61$ & $40.1 \pm 6.0$ \\
20 & $2.07 \pm 0.11$ & $42.13 \pm 2.89$ & $32.6 \pm 5.8$ \\
30 & $3.03 \pm 0.40$ & $44.90 \pm 3.06$ & $20.9 \pm 4.0$ \\
\hline
\end{tabular}




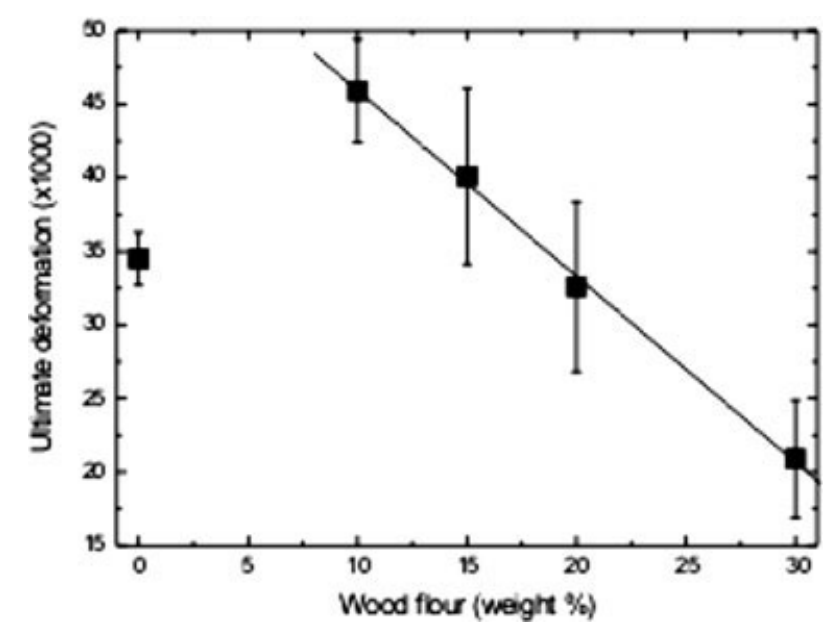

FIG. 4. Ultimate deformation as a function of the WF weight percentages.

while deformation at break decreases significantly $[8,20-$ 22]. In general, the reason for the failure of microcomposites at low strains is the initiation of the failure by interfacial debonding at multiple sites, which is followed by the coalescence of the cracks and final catastrophic crack growth $[9,23,24]$.

In our system, the mentioned failure mechanism is not present because of the excellent interfacial adhesion, which largely delays the final breakage of the material. Besides, the WF added to the composites and coreacted with the polyurethane can act as a chain extender, much in the same way as short diols added to segmented polyurethane formulations [9]. However, as more WF is added, the increased crosslinking and rigidity of the particles produce the overall traditional effect of increasing the composite modulus and reducing deformability of the material.

The experimental properties determined by tensile tests were compared with the predictions of simple theoretical models. The wood flour modulus was taken as $14 \mathrm{GPa}$ [25] and the modulus and strength of the matrix were experimentally determined $\left(E_{\mathrm{m}}=0.915 \mathrm{GPa} ; \sigma_{\mathrm{m}}=26\right.$ $\mathrm{MPa})$. The fitting parameters appearing in the Eqs. 1-4 were obtained from the nonlinear least squares regression based on the Levenberg-Marquardt algorithm. Figure 4 shows the experimental and calculated tensile modulus as a function of the WF content.

The series and parallel are the simplest models for a composite material of two components assuming that the individual phases are under uniform stress or strain, respectively. In all the range of compositions, the experimental values are between these two extreme limits, however, none of them are adequate to represent the modulus of these composites.

The experimental data fall on the curves predicted by the Hirsch and Halpsin-Tsai models (Fig. 5, curves 3 and 4 ), which give a good fit of the tensile modulus.

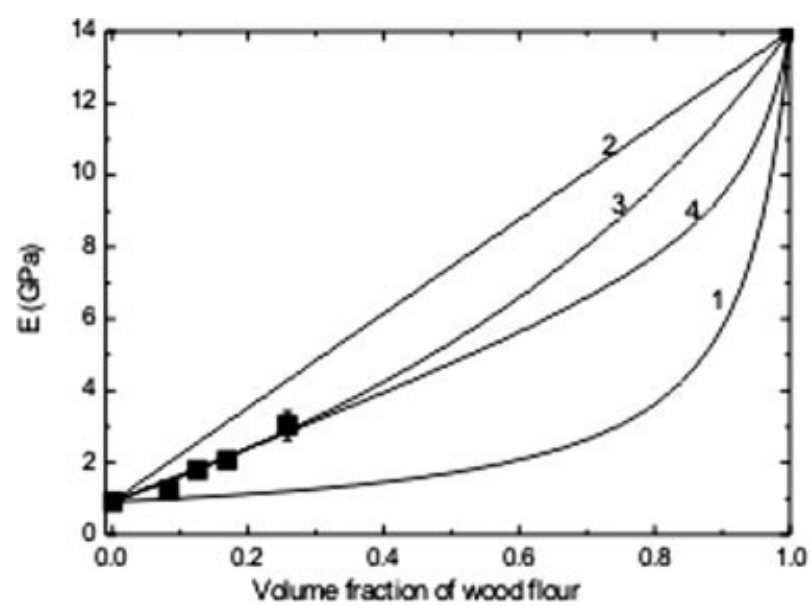

FIG. 5. Tensile modulus of reinforced polyurethanes and theoretical models (solid lines) as a function of WF concentration. Curve 1: series model; Curve 2: parallel model; Curve 3: Hirch model; Curve 4: Halpsin Tsai.

The Hirch parameters $x$ and $(1-x)$ are the proportions of material conforming to the upper and lower bound solutions. The best fitting of curve 3 was obtained with $x=$ 0.53 (for $x=0, E q .3$ reduces to the series model).

Halpsin-Tsai equation has one parameter, $\xi$, that serves to adjust the modulus between those of the fiber and the matrix. The best fitting was obtained with $\xi=13.9$.

From the analysis of the figure as well as the parameters calculated from the fitting procedures, it seems that although the fillers consist of irregular particles randomly dispersed in the matrix, the reinforcement is sensibly important. This again is the result of the very good interfacial adhesion and the additional crosslinking points in this region.

Figure 6 shows the ultimate stress of materials prepared with different percentages of $\mathrm{WF}$ and the fitting curve obtained using Eq. 6. All the composites present an ultimate stress higher than that of the neat matrix, which

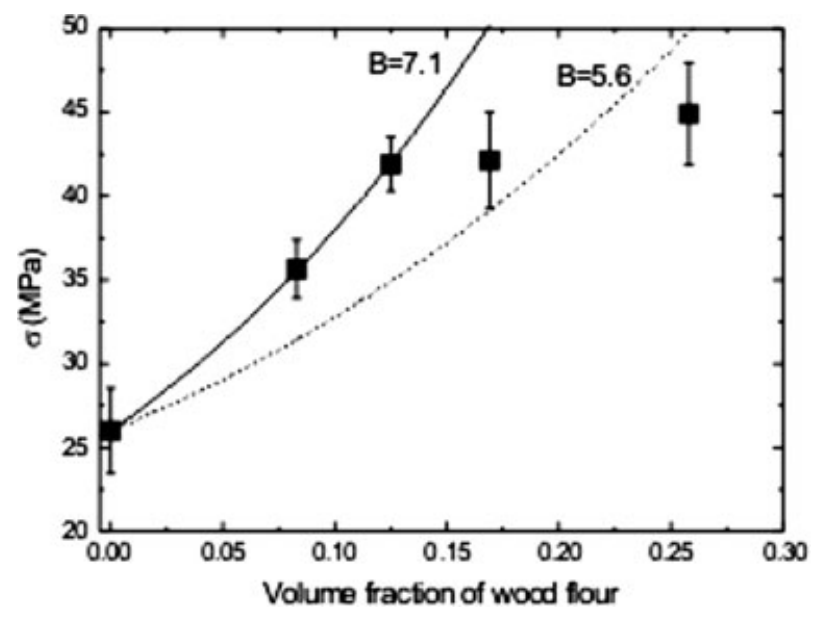

FIG. 6. Tensile strength of reinforced polyurethanes and Pukanzky model (solid line) as a function of WF volume fraction. 
TABLE 2. Impact properties of composites containing different weight percentages of wood flour.

\begin{tabular}{|c|c|c|c|c|c|}
\hline $\begin{array}{l}\text { Wood flour } \\
\text { (wt\%) }\end{array}$ & $E_{\mathrm{i}}(\mathrm{J} / \mathrm{m})$ & $E_{\mathrm{p}}(\mathrm{J} / \mathrm{m})$ & $E_{\mathrm{t}}(\mathrm{J} / \mathrm{m})$ & DI & $\sigma_{\mathrm{d}}(\mathrm{MPa})$ \\
\hline 0 & $24.4 \pm 4.1$ & $98.5 \pm 3.9$ & $122.8 \pm 6.6$ & $0.80 \pm 0.02$ & $164.9 \pm 25.0$ \\
\hline 10 & $29.3 \pm 1.9$ & $150.9 \pm 31.6$ & $180.2 \pm 32.8$ & $0.85 \pm 0.04$ & $229.0 \pm 21.9$ \\
\hline 20 & $35.4 \pm 2.6$ & $225.4 \pm 28.9$ & $260.7 \pm 29.0$ & $0.86 \pm 0.02$ & $260.8 \pm 28.4$ \\
\hline 30 & $33.0 \pm 1.9$ & $355.7 \pm 37.3$ & $388.7 \pm 36.3$ & $0.91 \pm 0.01$ & $279.3 \pm 36.2$ \\
\hline
\end{tabular}

means that the filler acts a reinforcing material. $B$ is a parameter that reflects the quality of the filler-matrix interface. According to D'Almeida and De Carvalho [26], for $B \leq 3$ no reinforcing effect is obtained. The solid curve in Fig. 6 was fitted using the experimental values of the samples with 0,10 , and $15 \mathrm{wt} \%$ of $\mathrm{WF}$, and thus, a $B$ parameter of 7.1 was obtained, which indicates that the interface is strong and a remarkable reinforcing effect is observed. However, the samples with 20 and $30 \mathrm{wt} \%$ of WF seem to deviate from the trend previously explained, behaving in a different way; even so, using all experimental values in the fitting process (dash line, Fig. 5) gives a $B$ parameter higher than $3(B=5.6)$.

In fiber-reinforced composites the composite strength is determined by the strength of the fiber and by the ability of the matrix to transmit stress to the fiber [15]. In particulate composites, if the particles are forming agglomerates in the composite, the transmission of stress to the particles (which is much less effective than transfer to fibers) is affected and the strength of the material is reduced. In this case, the formation of agglomerates was not observed by SEM, and therefore, this is another reason for the high ultimate stress observed in the reinforced polyurethanes. However, at high filler concentrations the ability of the matrix to transmit stress to the fibers could be reduced due to a dilution effect, i.e., the decreased proportion of matrix in the composite as compared with that of the particulate reinforcement.

\section{Impact Test}

The falling dart impact test is a convenient method used by the plastic industry to characterize the material response in the thickness direction [10].

The total impact energy can be divided into the elastic deformation energy $\left(E_{\mathrm{i}}\right)$ and the plastic deformation energy $\left(E_{\mathrm{p}}\right)$.

An ideal brittle material does not present any plasticity and $E_{\mathrm{t}}=E_{\mathrm{i}}$ and its ductile ratio is equal to zero. An ideal ductile material present $E_{\mathrm{t}} \gg>E_{\mathrm{i}}$ and its ductile index is close to 1 . A real polymer presents a ductile ratio between 0 and 1.

Table 2 shows the impact properties of the polyurethanes reinforced with $0,10,20$, and $30 \mathrm{wt} \%$ of $\mathrm{WF}$.

The impact strength $\left(\sigma_{\mathrm{d}}\right)$ shows a clear increase with WF concentration (about $70 \%$ with $30 \mathrm{wt} \%$ of WF). This behavior is related to the strong interface reinforcement- matrix and the generation of tortuous fracture paths through the WF-reinforced material.

The comparison between impact strength and tensile strength shows that the same trend can be observed in both properties (see Fig. 7). This behavior is attributed to the good interaction fiber-matrix that delays the failure of the material until the mechanical strength of the fiber combined with that of the polyurethane is reached.

The initiation energy slightly increases with the WF content, although it drops again at the highest WF concentration used (30 wt\%). However, the propagation energy and the total energy absorbed during the test increase significantly compared with that of the unreinforced polyurethane due to new mechanisms of energy dissipation that the filler introduces in the composites. The unreinforced polyurethane exhibits a less ductile behavior than that of the composites, with lower energy dissipation. This increase in ductile behavior related to the creation of larger surface areas during fracture is observed in the total and the propagation energies. Most of the energy of fracture is used in the propagation step. On the other hand, the increased crosslinking density of the composites already discussed, leads to an increased energy for crack initiation in the composites. However, as the WF concentration becomes high enough the material becomes more rigid and the energy required to initiate a crack drops, but since the filler still acts deflecting the crack paths, the total energy keeps increasing.

According to the above, the ductile index also increases with WF content indicating that the composites behave in a more ductile manner as compared with the matrix. The higher the DI value, the more energy is used

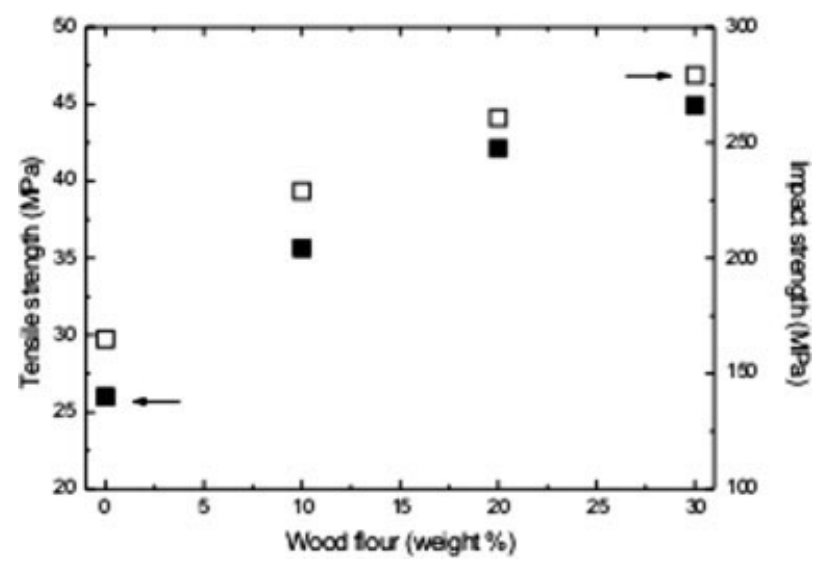

FIG. 7. Comparison between tensile and impact strength for the composites with different WF content. 
in propagating the failure in comparison to that used in crack initiation [27].

This is a very interesting result, since typically the addition of rigid fillers lead to the embrittlement of the material. In this case, however, the addition of WF to a compatible polyurethane matrix combined with the very good interfacial adhesion obtained, surprisingly leads to a stronger material that not only showed improved tensile strength, but also improved impact behavior.

\section{CONCLUSIONS}

Natural composites with excellent interface particlepolymer and uncommon high deformation at break and strength were prepared from wood flour and tung oil based-polyurethane.

Storage and tensile modulus increased with the WF content in the material. The strength also increased with WF concentration, denoting an excellent reinforcement effect of the WF in the composite material. The samples with up to $15 \mathrm{wt} \%$ of WF showed even higher ultimate deformation than the matrix. Moreover, no detriment in elongation at break was noticed for any of the composites studied.

The Hirsch and Halpin-Tsai models were adequate for modeling the dependence of the tensile modulus with WF concentration. The model used to predict the composite strength, in agreement with the SEM micrographs, indicates good compatibility between wood flour and polyurethane matrix.

Addition of wood flour has a beneficial effect on impact properties, concurring with tensile and DMA behavior.

\section{ACKNOWLEDGMENTS}

The authors thank National Research Council of Republic Argentina (CONICET) for the fellowship awarded to Dr. Mosiewicki.

\section{REFERENCES}

1. G. Woods, The ICI Polyurethanes Book, 2nd ed., Wiley, New York, Chapter 2 (1990).

2. K.S. Chian and L.H. Gan, J. Appl. Polym. Sci., 68, 509 (1998).

3. S.N. Khot, J.J. Lascala, E. Can, S.S. Morye, G.I. Williams, G.R. Palmese, S.H. Kusefoglu, and R.P. Wool, J. Appl. Polym. Sci., 82, 703 (2001).

4. Y.H. Hu, Y. Gao, D.N. Wang, C.P. Hu, S. Zhu, L. Vanoverloop, and D. Randall, J. Appl. Polym. Sci., 84, 591 (2002).

5. U. Casado, N.E. Marcovich, M.I. Aranguren, and M.A. Mosiewicki, "Tung Oil Based-polyurethanes Reinforced with Wood Flour," in 4th International Conference on Science and Technology of Composite Materials, Rio de Janeiro, Brazil, December 2007.
6. X. Kong and S.S. Narine, Industrial and Consumer Nonedible Products from Oils and Fats, Fereidoon Shahidi, Wiley, New York, Volume 6, Chapter 8, 279 (2005).

7. R.G. Kinabrew, Tung Oil in Mississippi, the Competitive Position of the Industry, University of Mississippi, University, MS (1952).

8. S. Demharter, J. Rösch, and R. Mülhaupt, Polym. Bull., 31, 421 (1993)

9. Q. Wu, M. Henriksson, X. Liu, and L.A. Berglund, Biomacromolecules, 8, 3687 (2007).

10. C.B. Bucknall and D.R. Paul, "Characterizing Toughness using Standard Empirical Tests," in Polymer Blends, C.B. Bucknall, Wiley, New York, Volume 2, 59 (2000).

11. G.I. Williams and R.P. Wool, Appl Compos. Mater., 7, 421 (2000).

12. S. Ahmed and F.R. Jones, J. Mater. Sci., 25, 4933 (1990).

13. N.E. Marcovich, M.M. Reboredo, and M.I. Aranguren, J. Appl. Polym. Sci., 70, 2121 (1998).

14. J.A. Manson and L.H. Sperling, Polymer Blends and Composites, 3rd ed., Plenum Press, New York, 373 (1981).

15. B. Pukanszky, F. Tudos, J. Jancar, and J. Kolarik, J. Mater. Sci. Lett., 8, 1040 (1989).

16. J. Urbanski, "Polyurethanes," in Handbook of Analysis of Synthetic Polymers and Plastics, J. Urbanski, W. Czerwinski, K. Janicka, F. Majewska, H. Zowall, Eds., Wiley, Polonia, 328 (1977). Chapter 11, Section 11.2 "Quantitative Analisis.”

17. B.L. Browning, Encyclopedia of Polymer Science and Technology, in: N.M. Bikales, N.G. Gaylord, H.F. Mark, Eds., Wiley, New York, Volume 15, 1 (1971).

18. N.E. Marcovich, M.M. Reboredo, and M.I. Aranguren, Polymer, 42, 815 (2001).

19. M.A. Mosiewicki, J. Borrajo, and M.I. Aranguren, Polym. Int., 54, 829 (2005).

20. M. Hiljanen-Vainio, M. Heino, and J.V. Seppälä, Polymer, 39, 865 (1998).

21. A. Torro-Palau, J.C. Fernandez-Garcia, A.C. Orgiles-Barcelo, M.M. Pastor-Blas, and J.M. Martin-Martinez, J. Adhesion Sci. Technol., 11, 247 (1997).

22. G. Woods. The ICI Polyurethanes Book, 2nd ed., Wiley, New York, Chapter 7 (1990).

23. D. Hull, Cambridge Solid State Science Series, Vol. 150: An Introduction to Composite Materials, 1 st ed., Cambridge University Press, Cambridge, 87 (1993).

24. B.A. Sjögren and L.A. Berglund, Polym. Compos., 20, 705 (1990)

25. D.N. Buttrey, in Polymer Engineering Composites, M.O.W. Richardson, Ed., Applied Science Publishers Ltd, London, 497 (1977).

26. J.R.M. D'Almeida and L.H. De Carvalho, J. Mater. Sci., 33, 2215 (1998).

27. N.L. Hancox, in Impact Behaviour of Fibre-Reinforced Composite Materials and Structures, Chapter 1, S.R. Reid and G. Zhou, Eds., CRC Press, Boca Raton, 2 (2001). 